\title{
113.
}

\section{NOTE ON THE GEOMETRICAL REPRESENTATION OF THE}

$$
\text { INTEGRAL } \int d x \div \sqrt{(x+a)(x+b)(x+c)} .
$$

[From the Philosophical Magazine, vol. v. (1853), pp. 281-284.]

THE equation of a conic passing through the points of intersection of the conics

$$
\begin{array}{r}
x^{2}+y^{2}+z^{2}=0, \\
a x^{2}+b y^{2}+c z^{2}=0,
\end{array}
$$

is of the form

$$
w\left(x^{2}+y^{2}+z^{2}\right)+a x^{2}+b y^{2}+c z^{2}=0,
$$

where $w$ is an arbitrary parameter. Suppose that the conic touches a given line, we have for the determination of $w$ a quadratic equation, the roots of which may be considered as parameters for determining the line in question. Let one of the values of $w$ be considered as equal to a constant quantity $k$, the line is always a tangent to the conic

$$
k\left(x^{2}+y^{2}+z^{2}\right)+a x^{2}+b y^{2}+c z^{2}=0 ;
$$

and taking $w=p$ for the other value of $w, p$ is a parameter determining the particular tangent, or, what is the same thing, determining the point of contact of this tangent.

The equation of the tangent is easily seen to be

$$
x \sqrt{b-c} \sqrt{a+k} \sqrt{a+p}+y \sqrt{c-a} \sqrt{b+k} \sqrt{b+p}+z \sqrt{a-b} \sqrt{c+k} \sqrt{c+p}=0 ;
$$

suppose that the tangent meets the conic $x^{2}+y^{2}+z^{2}=0$ (which is of course the conic corresponding to $w=\infty)$ in the points $P, P^{\prime}$, and let $\theta, \infty$ be the parameters of the point $P$, and $\theta^{\prime}, \infty$ the parameters of the point $P^{\prime}$, i.e. (repeating the defini- 
tion of the terms) let the tangent at $P$ of the conic $x^{2}+y^{2}+z^{2}=0$ be also touched by the conic $\theta\left(x^{2}+y^{2}+z^{2}\right)+a x^{2}+b y^{2}+c z^{2}=0$, and similarly for $\theta^{\prime}$. The coordinates of the point $P$ are given by the equations

$$
x: y: z=\sqrt{b-c} \sqrt{a+\theta}: \sqrt{c-a} \sqrt{b+\theta}: \sqrt{a-b} \sqrt{c+\theta} ;
$$

and substituting these values in the equation of the line $P P^{\prime}$, we have

$$
\begin{array}{r}
(b-c) \sqrt{a+k} \sqrt{a+p} \sqrt{a+\theta}+(c-a) \sqrt{b+k} \sqrt{b+p} \sqrt{b+\theta}+(a-b) \sqrt{c+k} \sqrt{c+p} \sqrt{c+\theta} \\
=0 \ldots\left(^{*}\right)
\end{array}
$$

an equation connecting the quantities $p, \theta$. To rationalize this equation, write

$$
\begin{aligned}
& \sqrt{(a+k)(a+p)(a+\theta)}=\lambda+\mu a, \\
& \sqrt{(b+k)(b+p)(b+\theta)}=\lambda+\mu b, \\
& \sqrt{(c+k)(c+p)(c+\theta)}=\lambda+\mu c,
\end{aligned}
$$

values which evidently satisfy the equation in question. Squaring these equations, we have equations from which $\lambda^{2}, \lambda \mu, \mu^{2}$ may be linearly determined; and making the necessary reductions, we find

$$
\begin{aligned}
\lambda^{2} & =a b c+k p \theta \\
-2 \lambda \mu & =b c+c a+a b-(p \theta+k p+k \theta), \\
\mu^{2} & =a+b+c+k+p+\theta
\end{aligned}
$$

or, eliminating $\lambda, \mu$,

$$
\{b c+c a+a b-(p \theta+k p+k \theta)\}^{2}-4(a+b+c+k+p+\theta)(a b c+k p \theta)=0,
$$

which is the rational form of the former equation marked $\left({ }^{*}\right)$. It is clear from the symmetry of the formula, that the same equation would have been obtained by the elimination of $L, M$ from the equations

$$
\begin{aligned}
& \sqrt{(k+a)(k+b)(k+c)}=L+M k, \\
& \sqrt{(p+a)(p+b)(p+c)}=L+M p, \\
& \sqrt{(\theta+a)(\theta+b)(\theta+c)}=L+M \theta ;
\end{aligned}
$$

and it follows from Abel's theorem (but the result may be verified by means of Euler's fundamental integral in the theory of elliptic functions), that if

$$
\Pi x=\int_{\infty} \frac{d x}{\sqrt{(x+a)(x+b)(x+c)}},
$$

then the algebraical equations $\left({ }^{*}\right)$ are equivalent to the transcendental equation

$$
\pm \Pi k \pm \Pi p \pm \Pi \theta=0
$$


the arbitrary constant which should have formed the second side of the equation having been determined by observing that the algebraical equation gives for $p=\theta$, $k=\infty$, a system of values, which, when the signs are properly chosen, satisfy the transcendental equation. In fact, arranging the rational algebraical equation according to the powers of $k$, it becomes

$$
\begin{aligned}
& k^{2}(p-\theta)^{2}-2 k\{p \theta(p+\theta)+2(a+b+c) p \theta+(b c+c a+a b)(p+\theta)+2 a b c\} \\
+ & p^{2} \theta^{2}-2(b c+c a+a b) p \theta-4 a b c(p+\theta)+b^{2} c^{2}+c^{2} a^{2}+a^{2} b^{2}-2 a^{2} b c-2 b^{2} c a-2 c^{2} a b=0 ;\left(^{*}\right)
\end{aligned}
$$

which proves the property in question, and is besides a very convenient form of the algebraical integral. The ambiguous signs in the transcendental integral are not of course arbitrary (indeed it has just been assumed that for $p=\theta, \Pi p$ and $\Pi \theta$ are to be taken with opposite signs), but the discussion of the proper values to be given to the ambiguous signs would be at all events tedious, and must be passed over for the present.

It is proper to remark, that $\theta=p$ gives not only, as above supposed, $k=\infty$, but arother value of $k$, which, however, corresponds to the transcendental equation

$$
\pm \Pi k \pm 2 \Pi p=0 ;
$$

the value in question is obviously

$$
4 k=\frac{p^{4}-2(b c+c a+a b) p^{2}-8 a b c p+b^{2} c^{2}+c^{2} a^{2}+a^{2} b^{2}-2 a^{2} b c-2 b^{2} c a-2 c^{2} a b}{(p+a)(p+b)(p+c)} .
$$

Consider, in general, a cubic function $\mathrm{a} x^{3}+3 \mathrm{~b} x^{2} y+3 \mathrm{c} x y^{2}+\mathrm{d} y^{3}$, or, as I now write it in the theory of invariants, $(\mathrm{a}, \mathrm{b}, \mathrm{c}, \mathrm{d})(x, y)^{3}$, the Hessian of this function is

$$
\left(a c-b^{2}, \frac{1}{2}(a d-b c), b d-c^{2}\right)(x, y)^{2},
$$

and applying this formula to the function $(p+a)(p+b)(p+c)$, it is easy to write the equation last preceding in the form

$$
4 k=p-(a+b+c)-\frac{9 \text { Hessian }\{(p+a)(p+b)(p+c)\}}{(p+a)(p+b)(p+c)},
$$

which is a formula for the duplication of the transcendent $\Pi x$.

Reverting now to the general transcendental equation

$$
\pm \Pi k \pm \Pi p \pm \Pi \theta=0,
$$

we have in like manner

$$
\pm \Pi k \pm \Pi p \pm \Pi \theta^{\prime}=0 ;
$$

and assuming a proper correspondence of the signs, the elimination of $\Pi p$ gives

$$
\Pi \theta^{\prime}-\Pi \theta=2 \Pi k
$$


i.e. if the points $P, P^{\prime}$ upon the conic $x^{2}+y^{2}+z^{2}=0$ are such that their parameters $\theta, \theta^{\prime}$ satisfy this equation, the line $P P^{\prime}$ will be constantly a tangent to the conic

$$
k\left(x^{2}+y^{2}+z^{2}\right)+\left(a x^{2}+b y^{2}+c z^{2}\right)=0 .
$$

Hence also, if the parameters $k, k^{\prime}, k^{\prime \prime}$ of the conics

$$
\begin{aligned}
& k\left(x^{2}+y^{2}+z^{2}\right)+a x^{2}+b y^{2}+c z^{2}=0 \\
& k^{\prime}\left(x^{2}+y^{2}+z^{2}\right)+a x^{2}+b y^{2}+c z^{2}=0 \\
& k^{\prime \prime}\left(x^{2}+y^{2}+z^{2}\right)+a x^{2}+b y^{2}+c z^{2}=0
\end{aligned}
$$

satisfy the equation

$$
\Pi k+\Pi k^{\prime}+\Pi k^{\prime \prime}=0,
$$

there are an infinity of triangles inscribed in the conic $x^{2}+y^{2}+z^{2}=0$, and the sides of which touch the last-mentioned three conics respectively.

Suppose $2 \Pi k=\Pi \kappa$ (an equation the algebraic form of which has already been discussed), then

$$
\Pi \theta^{\prime}-\Pi \theta=\Pi_{\kappa},
$$

$\theta=\infty$ gives $\theta^{\prime}=\kappa$; or, observing that $\theta=\infty$ corresponds to a point of intersection of the conics $x^{2}+y^{2}+z^{2}=0, a x^{2}+b y^{2}+c z^{2}=0, \kappa$ is the parameter of the point in which a tangent to the conic $k\left(x^{2}+y^{2}+z^{2}\right)+a x^{2}+b y^{2}+c z^{2}=0$ at any one of its intersections with the conic $x^{2}+y^{2}+z^{2}=0$ meets the last-mentioned conic. Moreover, the algebraical relation between $\theta, \theta^{\prime}$ and $\kappa$ (where, as before remarked, $\kappa$ is a given function of $k$ ) is given by a preceding formula, and is simpler than that between $\theta, \theta^{\prime}$ and $k$.

The preceding investigations were, it is hardly necessary to remark, suggested by a well-known memoir of the late illustrious Jacobi, and contain, I think, the extension which he remarks it would be interesting to make of the principles in such memoir to a system of two conics. I propose reverting to the subject in a memoir to be entitled "Researches on the Porism of the in- and circumscribed triangle." [This was, I think, never written.] 